\title{
Axillary approach versus the infraclavicular approach in ultrasound-guided brachial plexus block: comparison of anesthetic time
}

\author{
In Ae Song ${ }^{1}$, Nam-Su Gill ${ }^{2}$ Eun-young $\mathrm{Choi}^{3}$, Sung-Eun $\mathrm{Sim}^{2}$, Seong-Won $\mathrm{Min}^{2}$, Young-Jin $\mathrm{Ro}^{4}$, and \\ Chong Soo Kim ${ }^{2}$ \\ Department of Anesthesiology and Pain Medicine, ${ }^{1}$ Seoul National University Bundang Hospital, ${ }^{2}$ Boramae Municipal Hospital, \\ ${ }^{3}$ Seoul National University Hospital, Seoul National University College of Medicine, ${ }^{4}$ Asan Medical Center, University of Ulsan College \\ of Medicine, Seoul, Korea
}

Background: With ultrasound guidance, the success rate of brachial plexus block (BPB) is $95-100 \%$ and the anesthetic time has become a more important factor than before. Many investigators have compared ultrasound guidance with the nerve stimulation technique, but there are few studies comparing different approaches via the same ultrasound guidance. We compared the axillary BPB with the infraclavicular BPB under ultrasound guidance.

Methods: Twenty-two ASA physical status I-II patients presenting with elective forearm surgery were prospectively randomized to receive an axillary BPB (group AX) or an infraclavicular BPB (group IC) with ultrasound guidance. Both groups received a total of $20 \mathrm{ml}$ of $1.5 \%$ lidocaine with $5 \mu \mathrm{g} / \mathrm{ml}$ epinephrine and $0.1 \mathrm{mEq} / \mathrm{ml}$ sodium bicarbonate. Patients were then evaluated for block onset and block performance time was also recorded.

Results: Group IC demonstrated a reduction in performance time vs. group AX (622 $\pm 139 \mathrm{sec}$ vs. $789 \pm 131 \mathrm{sec}, \mathrm{P}<$ $0.05)$. But, the onset time was longer in group IC than in group $\mathrm{AX}(7.7 \pm 8.8 \mathrm{~min}$ vs. $1.4 \pm 2.3 \mathrm{~min}, \mathrm{P}<0.05)$. All blocks were successful in both groups.

Conclusions: Under ultrasound guidance, infraclavicular BPB was faster to perform than the axillary approach. But the block onset was slower with the infraclavicular approach. (Korean J Anesthesiol 2011; 61: 12-18)

Key Words: Brachial plexus, Nerve block, Ultrasound.

Received: November 4, 2010. Revised: 1st, November 25, 2010; 2nd, February 18, 2011. Accepted: February $24,2011$.

Corresponding author: Chong Soo Kim, M.D., Department of Anesthesiology and Pain Medicine, Boramae Municipal Hospital, 39, Boramaegil, Dongjak-gu, Seoul 156-707, Korea. Tel: 82-2-870-2511, Fax: 82-2-846-2985, E-mail: cskim@brm.co.kr

(ㄷ) This is an open-access article distributed under the terms of the Creative Commons Attribution Non-Commercial License (http:// creativecommons.org/licenses/by-nc/3.0/), which permits unrestricted non-commercial use, distribution, and reproduction in any medium, provided the original work is properly cited. 


\section{Introduction}

The brachial plexus block (BPB) is routinely performed since it is as effective as general anesthesia in anesthetizing the upper limbs and is less invasive. As with other areas in medicine, the technique for the BPB has been improved upon by the development of medical technology. Early on, local anesthesia was injected in an area near on the axillary artery or by paresthesia experienced by the patient. However, with the introduction of a nerve stimulator, the previous non-visual approach was technologically advanced. Now, anestheologists did not have to rely on subjective symptoms given by the patient and they can objectively use signals from muscle contractions to perform blocks. Recently, as ultrasound equipment became widely available, the use of the equipment for nerve blocks became easier for anesthetists and the non-visual method performed in nerve blocks developed into a visual method.

Similar to the nerve stimulator guided technique, an ultrasound-guided BPB can be performed in various places such as the axillar, supraclavicular, infraclavicular, and interscalene areas. There have been many reports comparing various methods for nerve stimulator guided technique $[1,2]$. However, there are few reports regarding techniques using the ultrasound-guided method. Many researchers have compared the ultrasound-guided technique to the nerve stimulator guided technique, but there are not many comparative studies between the various methods for ultrasound-guided nerve blocks $[3,4]$.

When comparing the methods for the $\mathrm{BPB}$, the success rate has been reported as the most important indicator. However, with the introduction of the ultrasound-guided technique, the success rate has reached $95-100 \%[5,6]$. Therefore, a different indicator is required in comparative studies today, and the anesthetic time is considered as an important indicator after the success rate [1]. When the anesthetic time is delayed, the turnover ratio of operating rooms decreases and the inconvenience the patients are subjected to increases. Anesthetic time is the sum of the performance time and onset time. Contrary to the onset time, the performance time also inconveniences the patients. Therefore, the primary aim of this study was to determine the performance time with the onset time and anesthetic time being measured at the same time.

A helpful way to reduce performance time is to simplify the procedure. Ultrasound-guided axillary block requires two to three needle insertions, whereas ultrasound-guided infraclavicular block requires only one needle insertion and one injection of local anesthesia [7]. Hence, the authors conducted a randomized controlled clinical trial with the hypothesis that the performance time of an ultrasound-guided infraclavicular block will be shorter compared to an ultrasound-guided axillary block.

\section{Meterials and Methods}

The study was approved by the institutional review board and informed consent being obtained after a detailed explanation of the purpose of the study as well as the risks and complications of the procedure were explained to the patients. This study was done on ASA physical status I-II patients between $18-$ 80 years of age scheduled for forearm surgery at our hospital from February to May of 2010. Patients with paresthesia or paresis in an operating upper limb were excluded from the study. In addition, cases where BPB was refused and cases where there were constraints in the local area of anesthesia such as coagulopathy, local infection in the area of the block and hypersensitivity for local anesthesia were also excluded. Twenty-two patients were randomized to receive an axillary BPB (Group AX, $\mathrm{n}=11$ ) or an infraclavicular BPB (Group IC, $\mathrm{n}=$ 11). Randomization was done using a randomization program on the Internet (http://www.randomization.com/).

Without any premedication, the patients were transferred to a room to perform the block (i.e. not the operating room), and the vitals were monitored by noninvasive blood pressure monitoring, pulse oximeter, and electrocardiogram. Both groups underwent ultrasound-guided nerve block using the inplane technique and all block procedures were performed by one researcher. This researcher has done more than 20 cases each for both the ultrasound-guided axillary approach and the infraclavicular approach. Both groups received $20 \mathrm{ml}$ of $1.5 \%$ lidocaine with epinephrine $(1: 200,000)$ and $0.1 \mathrm{mEq} / \mathrm{ml}$ of sodium bicarbonate.

Patients in Group AX were laid in the supine position with the arm to be blocked externally rotated more than 90 degrees and the elbow flexed to expose the armpit. Betadine was applied to the skin of the armpit and all procedures were done using sterile technique. A 13-6 MHz probe (SLA, Sonosite, USA) for ultrasound (Micromaxx ${ }^{\circledR}$, Sonosite, USA) was positioned in the axillary crease perpendicular to the axillary artery for observation of the axillary artery and surrounding structures. The axillary artery was confirmed with color Doppler image. A $50 \mathrm{~mm} 22$ gauge Sprotte needle (Nanoline ${ }^{\circledR}$, PAJUNK, Germany) was inserted using real time ultrasound guidance to infiltrate the right, left, and back of the axillary artery with anesthetic medication. First the block needle was inserted in-plane above the ultrasound probe for injection of $5 \mathrm{ml}$ of the anesthetic to the back of the axillary artery. Here, it was confirmed that the block medication did not spread under the triceps fascia behind the axillary artery. Then, $5 \mathrm{ml}$ of the anesthetic was injected to the side of the axillary artery. The tip of the needle was inserted in close proximity to the axillary artery slightly pushing without puncturing the axillary artery, and the infiltration of the local anesthesia was confirmed to be spread around the artery and 
not toward the surrounding muscle. Next, the block needle was removed and re-inserted in-plane below the ultrasound probe for injection of $5 \mathrm{ml}$ of the anesthetic to the side of the axillary artery. Finally, the coracobrachialis and biceps were observed with the ultrasound probe to find the musculocutaneous which runs between the coracobrachialis or coracobrachialis and biceps. Then $5 \mathrm{ml}$ of anesthetic was infiltrated around the nerve. While performing the axillary approach, the block needle was inserted into the skin twice during the infiltration around the axillary artery and once for blocking of the musculocutaneous (a total of three injections).

The patients in Group IC were laid in a supine position with their arms beside their body with only the head turned toward the opposite direction of the block area. Betadine was applied to the skin on the infraclavicular area and all procedures were performed using sterile technique. The axillary artery was found using the 13-6 MHz ultrasound probe (SLA, Sonosite, USA) in the infraclavicular fossa and the probe was positioned perpendicular to the axillary artery. The artery was confirmed using color Doppler, and after local anesthesia, an $80 \mathrm{~mm}$ 18 gauge Tuohy needle (Perican ${ }^{\circledR}$, B. Braun, Germany) was inserted above the ultrasound probe. The needle tip was positioned at the bottom of the axillary artery (patient's back area) and $2 \mathrm{ml}$ of anesthetic was injected. After confirming the "double bubble sign" where hypoechoic bubbles appear due to the medication, the remaining $18 \mathrm{ml}$ was injected [7].

The duration of each procedure was measured from the time the Betadine was applied to the skin to the end of the infiltration of the anesthetic, including the removal of the block needle. The nerve block of the patient was evaluated immediately after the block procedure and at 5, 10, 15, 20, and 30 minutes afterwards. The sensory nerves were assessed by alcohol swab testing the radial nerve (posterior part of wrist and of the three first fingers), median nerve (anterior part of wrist and of the three first fingers), ulnar nerve (medial part of wrist and of the hand), musculocutaneous nerve (lateral part of forearm), axillary nerve (shoulder), medial brachial nerve (medial part of arm) and medial antebrachial nerve (medial part of forearm): responses were compared with the opposite corresponding areas. No sensation was recorded as 0 ; hypothesia was recorded as 1 , and normal sensation was recorded as 2 . In this method, when hypoesthesia occurred in the radial, median, ulnar and musculocutaneous nerves, this was defined as the start of the nerve block onset time. Regarding motor nerves, the radial (elbow extension), median (third finger flexion), ulnar (fifth finger flexion), musculocutaneous (elbow flexion) and axillary nerves (arm abduction) were assessed with normal muscle power recorded as 5; slightly reduced power recorded as 4; significant reduction in power recorded as 3; unable to move against gravity or only contract the muscles recorded as
2 , and complete paralysis was recorded as 1 . The nerve block onset time of the motor nerves was defined as when the radial, median, ulnar, and musculocutaneous nerves scored lower than 3 . In the case when there was no onset of nerve block 30 minutes after the procedure, it was considered as a block failure and an additional block was performed. In addition, cases where general anesthesia was administered due to pain during surgery were also recorded as a block failure. The occurrence of complications such as hemorrhage, hematoma, pneumothorax, and intravascular injection were also recorded.

Using the results from an existing study that compared ultrasound-guided infraclavicular block to nerve stimulator guided axillary block, the sample size necessary for a 0.8 statistical power and 0.05 type I error was calculated to be 11 per group [7]. In the statistical analysis, the continuous variable was expressed as the mean \pm standard deviation and a Student's t-test was used for the analysis. The categorical variables were analyzed using a Fisher's exact test. SPSS (ver 12.0, SPSS Inc, USA) was used as the statistics program and differences were considered statistically significant when the $P$ value was less than 0.05 .

\section{Results}

There were no significant differences between the groups for age, sex, height, weight and physical status (Table 1). The block was successful in all patients for both groups so there were no cases needing an additional block or general anesthesia. Hypoesthesia in the axillary nerve occurred in 2 patients (18\%) in Group AX and in 9 patients (82\%) in Group IC. The duration of the block procedure was notably shorter for Group IC (622 \pm $139 \mathrm{sec})$ compared to Group AX $(789 \pm 131 \mathrm{sec})(\mathrm{P}<0.05$, Fig. 1). On the contrary, the onset time of the block was notably faster for Group AX (1.4 $\pm 2.3 \mathrm{~min})$ than for Group IC (7.7 $\pm 8.8 \mathrm{~min})$ $(\mathrm{P}<0.05)$. As a result, there were no significant differences between the two groups (Group AX, $870 \pm 193 \mathrm{sec}$ vs. Group IC $1,085 \pm 526 \mathrm{sec}$ ) for the total anesthetic time which included the performance time and the onset time.

Table 1. Demographic Data

\begin{tabular}{lccc}
\hline & $\begin{array}{c}\text { Group AX } \\
(\mathrm{n}=11)\end{array}$ & $\begin{array}{c}\text { Group IC } \\
(\mathrm{n}=11)\end{array}$ & P value \\
\hline Age $(\mathrm{yr})$ & $49.5 \pm 17.5$ & $37.9 \pm 17.6$ & 0.139 \\
Sex $(\mathrm{M} / \mathrm{F})$ & $8 / 3$ & $8 / 3$ & 1.000 \\
Weight $(\mathrm{kg})$ & $72.1 \pm 12.5$ & $64.2 \pm 13.0$ & 0.162 \\
Height $(\mathrm{cm})$ & $166.2 \pm 10.4$ & $166.8 \pm 9.0$ & 0.888 \\
ASA physical status (I/II) & $7 / 4$ & $9 / 2$ & 0.635 \\
\hline
\end{tabular}

Data are expressed as means \pm SD or number of patients. AX: axillary approach, IC: infraclavicular approach, ASA: American Society of Anesthesiologists. 


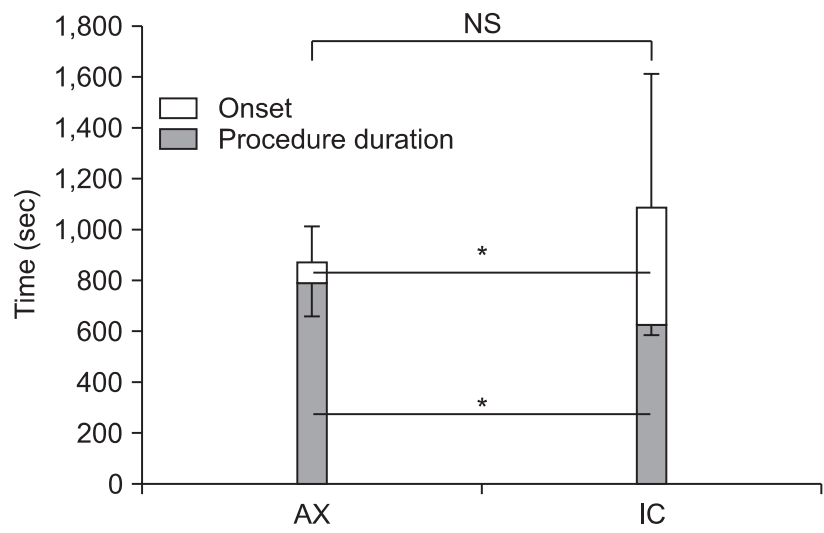

Fig. 1. Total anesthetic time, onset and procedure duration of the axillary approach (AX) and the infraclavicular approach (IC). Total anesthetic time is the sum of the onset and the procedure duration. The procedure duration was significantly shorter in the IC group. The onset is significantly shorter in AX group. As a result, the total anesthetic time was similar between two groups. NS: not significant. $* \mathrm{P}<0.05$.
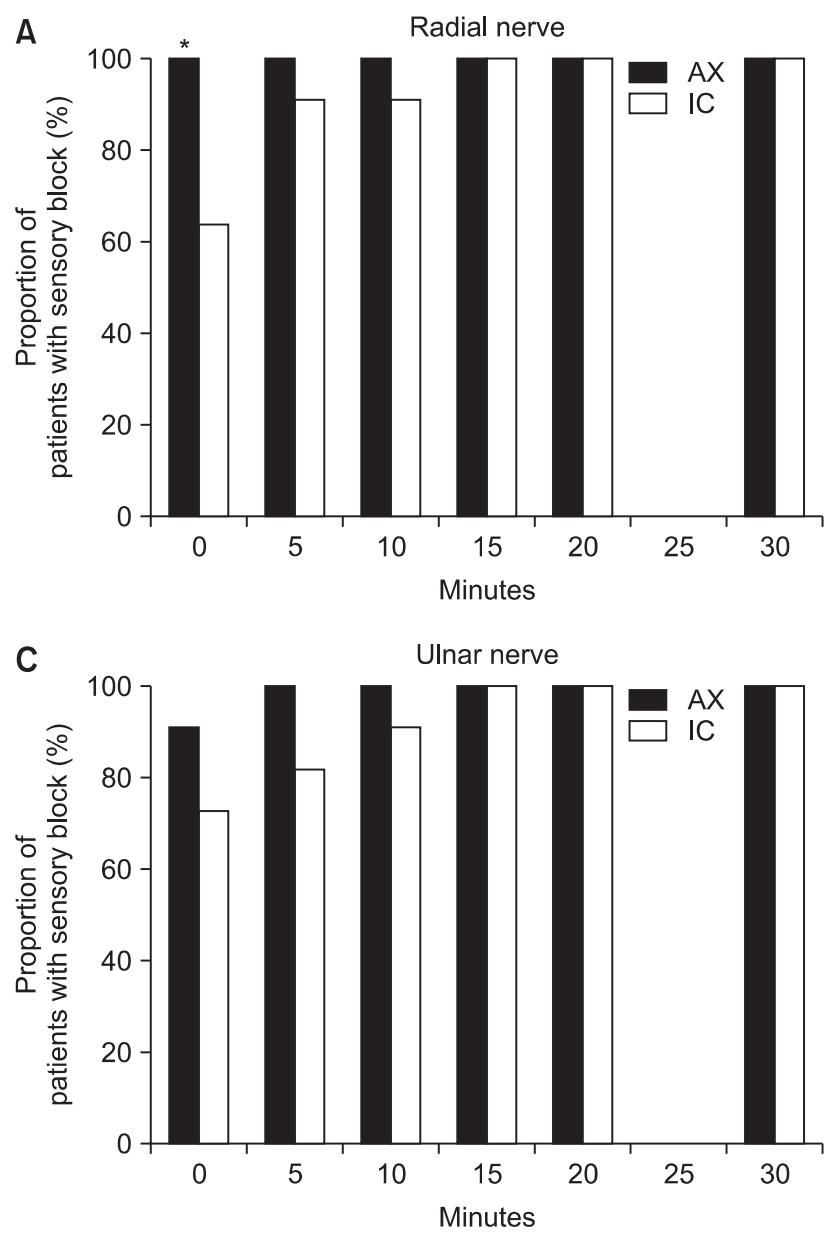

Fig. 2 and 3 compare the onset of the sensory block or motor block in patients according to each time bracket. Although a higher rate was consistently seen for patients in Group AX with the sensory block and for patients in Group IC with the motor block excluding the musculocutaneous nerve, there were no significant differences other than in the radial sensory nerve immediately after the block (Fig. 2A). The ratio of Group AX patients with a motor block of the musculocutaneous nerve was higher and there was a significant difference immediately after and 5 minutes after the block (Fig. 3D). There were no complications of hemorrhage, hematoma, pneumothorax, or intravascular injection for both groups.

\section{Discussion}

Results of this prospective randomized comparative study, demonstrated that ultrasound-guided infraclavicular BPB had a shorter anesthetic performance time than the axillary approach.
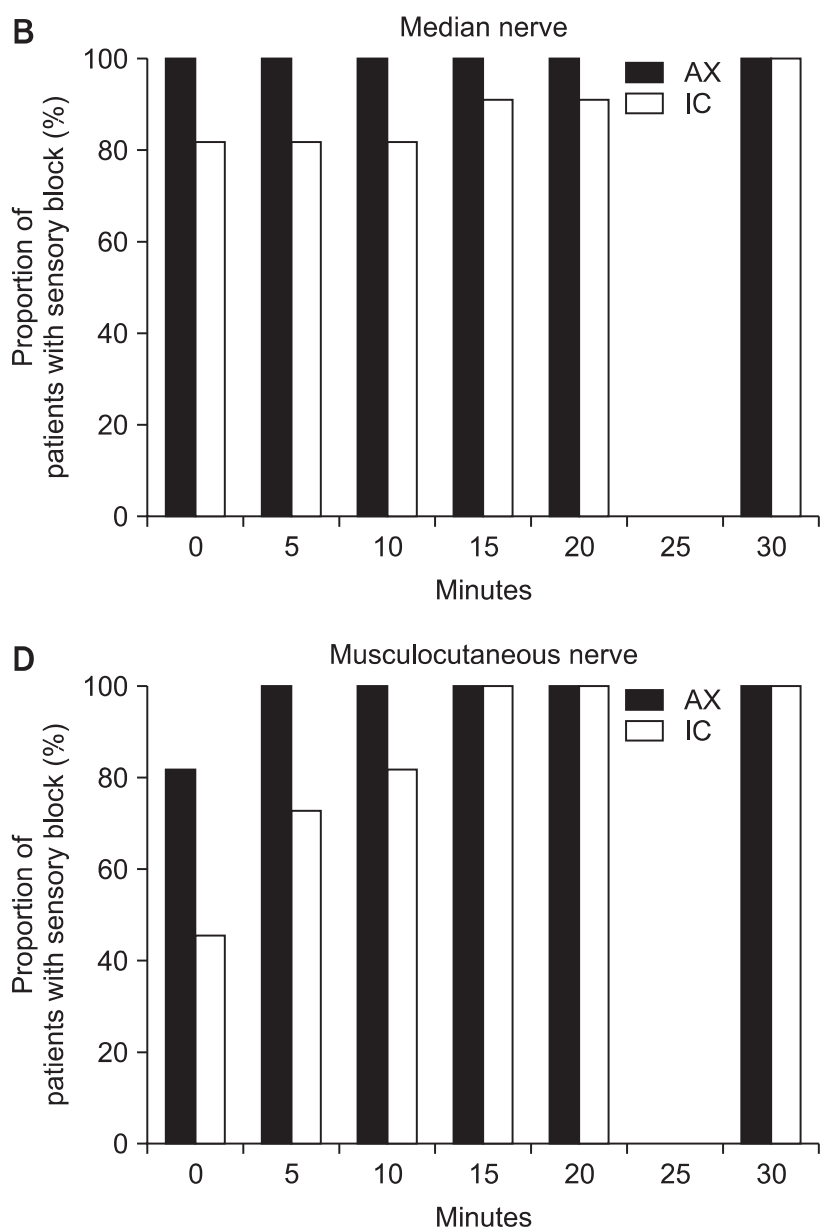

Fig. 2. Percentage of patients with sensory block (score of 1-0) according to the time of cutaneous distribution of: (A) the radial nerve, (B) the median nerve, (C) the ulnar nerve and (D) the musculocutaneous nerve. Significantly more patients showed sensory block of the radial nerve in the AX group immediately after block procedure. AX: Axillary approach group, IC: Infraclavicular approach group. Sensory score: 0 , no sensation; 1 , hypoesthesia; 2 , normal sensation. ${ }^{*} \mathrm{P}<0.05$ compared with IC group. 
A
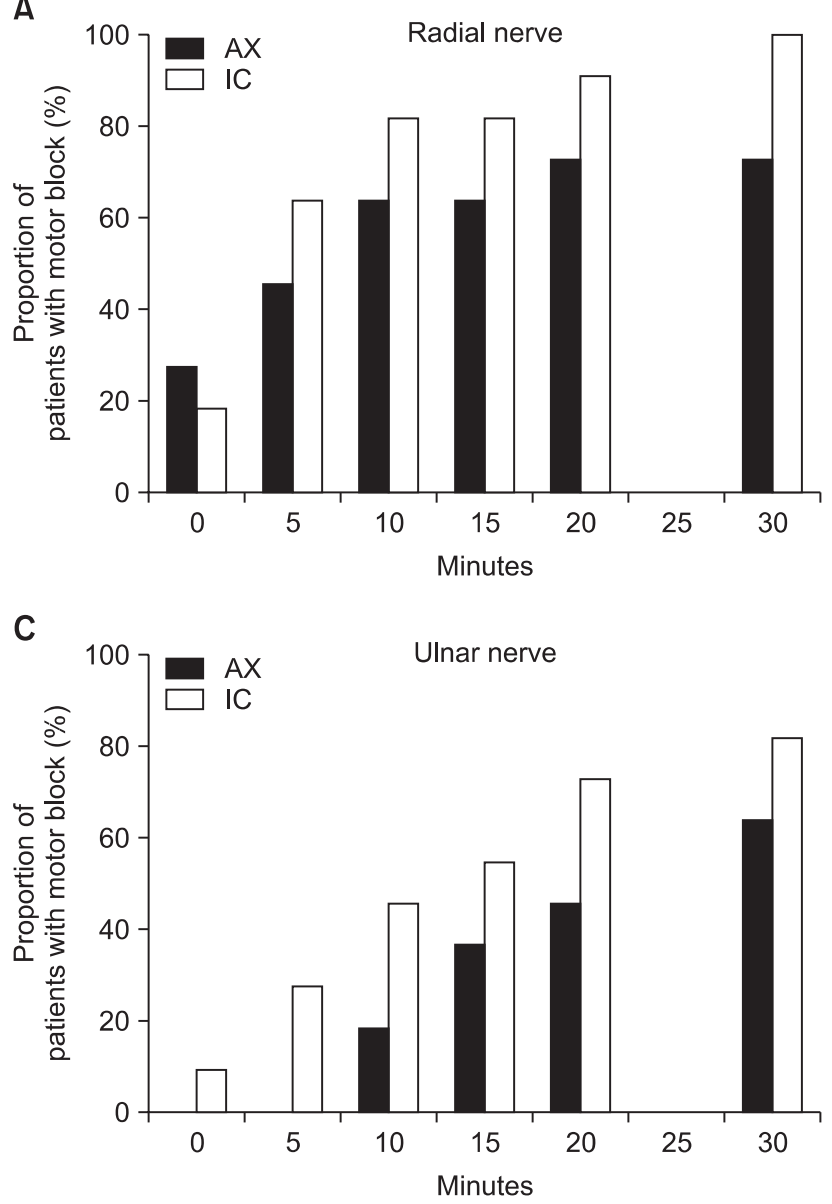

B

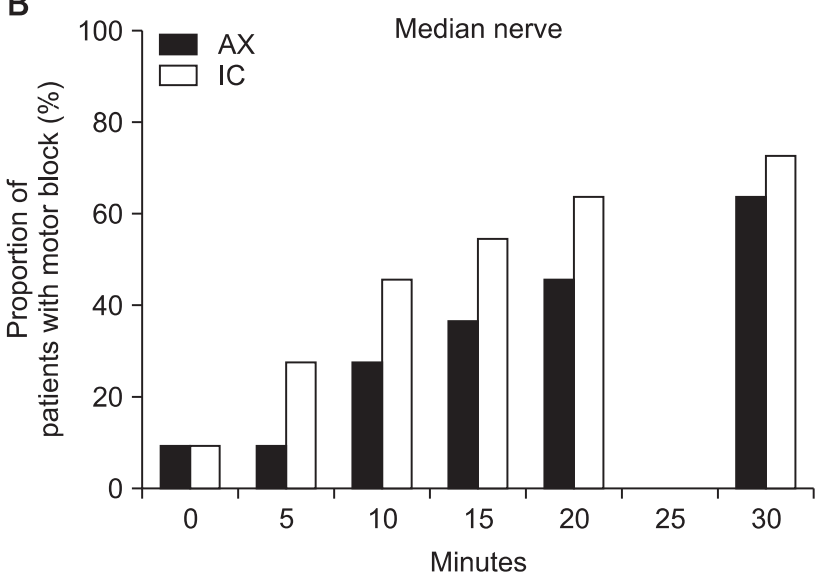

D

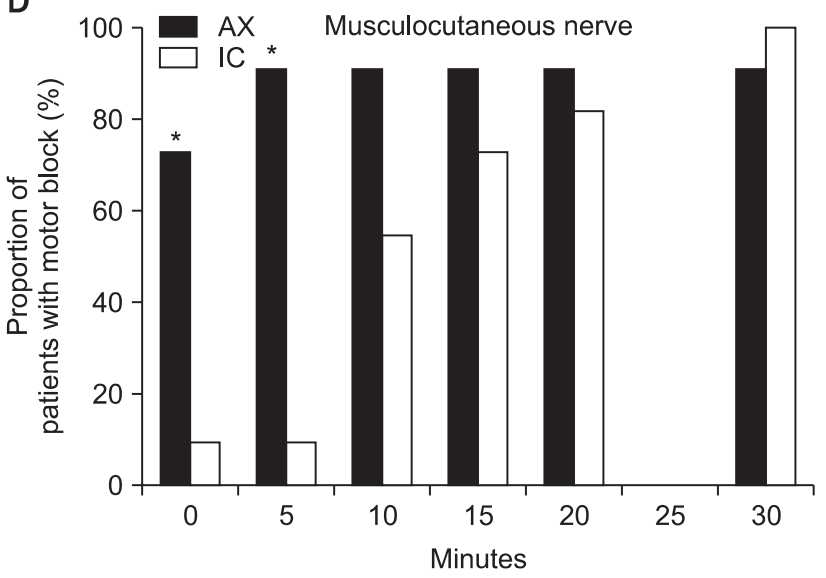

Fig. 3. Percentage of patients with motor block (score of 2-1) according to the time of cutaneous distribution of: (A) the radial nerve, (B) the median nerve, (C) the ulnar nerve and (D) the musculocutaneous nerve. Significantly more patients showed motor block of musculocutaneous nerve in the AX group 0 and 5 minutes after block procedure. AX: Axillary approach group, IC: Infraclavicular approach group. Motor score: 1, complete paralysis; 2 , inability to move against gravity; 3 , significant muscle strength reduction; 4 , slightly reduced muscle strength; 5 , normal muscle strength. ${ }^{*} \mathrm{P}<0.05$ compared with IC group.

Since there were no block failures in both groups, there were no differences found in the success rate. The difference in the performance time was because the infraclavicular approach required only one injection of local anesthetic while the axillary approach requires three around the axillary nerve and one in the musculocutaneous nerve area for a total of four injections. In our study, there was approximately a 3 minute difference in the performance time and this reduction in the performance time is more convenience and comfortable for the patient who needs to be under a drape for the procedure. Moreover, a reduction in the performance time also provides time for the anesthetist to focus on the patient and other matters. In contrast to the performance time, the onset time of the infraclavicular approach appeared to be longer. However, there was no significant difference when the performance time and onset time were added together for the total anesthetic time. These results concur with existing research that compared the infraclavicular approach and the humeral approach using a nerve stimulator [1]. The reason that the onset time is different is thought to be that in the axillary approach infiltration of the local anesthesia is done at the periphery of the nerve while the infraclavicular approach, local anesthetic is sprayed in the proximal area of the nerve. In addition, in the axillary approach, onset may begin during the infiltration process of the four local anesthetics so the onset time may be recorded shorter. The longer onset time could be a shortcoming of the infraclavicular approach, but in actual procedures, preparations such as draping of the skin can begin after the block has started to progress, and in orthopedic surgery where infection must be avoided, it takes a long time to drape and prepare the skin so the block can progress during this time. In addition to the fact that the infraclavicular approach only requires one injection of the local anesthesia, there is also no need to abduct the patient's arm. This is very important for patients who cannot 
move their arm or shoulder due to injury or fracture. When the rate of patients blocked at each time point was compared to the observed progress of the block, there were notable differences in only three areas (Fig. 2 and 3). Especially in the motor block of the musculocutaneous nerve, there were large differences immediately after block and after 5 minutes because in Group AX, the musculocutaneous nerve was found to specifically infiltrate local anesthesia directly around the nerve. This result suggests that infiltration of the nerve directly with local anesthesia has a faster onset time than infiltrating around the artery. In Group AX, there was no need to find each structure in the block of the radial, median and ulnar nerves since the aim was to block through injection of the arterial area [6].

In this study the performance time was defined as from the time Betadine was applied onto the skin to the removal of the block needle. This was defined differently in other studies where it was measured needle to needle $[1,6-8]$, and this was to measure the more realistic time to induce $\mathrm{BPB}$ anesthesia. Of course, the time needed to apply the Betadine to the skin and prepare the area may affect the results, but since all the procedures were done by one researcher, the time was considered to be consistent. Therefore, this study showed a decrease in the actual time to anesthetize a patient. However, not measuring the actual performance time from needle insertion to removal can be a limitation of this study.

The onset time of the nerve block was defined as when hypothesia occurred in the dermatome of the four nerves. This is also different from previous studies which were based on a complete loss of sensation $[1,6-8]$. The reason for this is when a complete loss of sensation is the standard, the onset time becomes delayed by more than 30 minutes after the treatment, which is beyond the scope of the measurement. There are two reasons for this. First, both groups used a relatively small amount of the local anesthesia $(20 \mathrm{ml})$. The authors wanted to avoid complications from overmedication in the infraclavicular $\mathrm{BPB}$, which they had relatively little experience with. Therefore, $20 \mathrm{ml}$ was chosen, which is the lowest amount advised in theory for infraclavicular BPB [2]. In addition, to eliminate any confounding factor from the dosage of the anesthetics, the same amount was used in the axillary approach. It was confirmed from preliminary research that such small amounts do not interfere with the anesthesia and surgery. However, in this study, it delayed the progression of the nerve block. Of course, there were no disruptions in the anesthesia or operations in this study, but there were difficulties when comparing the onset time. The second factor is that an alcohol swab test was used rather than a pinprick test to measure sensory function. The authors thought that the pinprick test, which is not commonly used in actual situations, can be uncomfortable for the patient; therefore, it was substituted with the alcohol swab test. However, the alcohol swab test has the problem that even when thermal sense is completely lost, a weak contact can record sensory function at a score of 1 . This can be improved if a scoring system that separates thermal sense and sense of touch is used.

In this study, an 18 gauge Tuohy needle was used as the block needle when performing the infraclavicular approach. In the infraclavicular approach, the target location was deep; thus, a general block needle was not very visible in the ultrasound imaging. In addition to the approach having a deeper target, the needle has to be much more vertical which also lowers the visibility. Hence, the epidural Tuohy needle was chosen since it is a thick needle with a needle tip that causes the least amount of damage to the nerves. Although a thick needle was used, sufficient local anesthesia was administered before insertion to greatly reduce the patient's discomfort. An echogenic needle, which is highly visible in ultrasound, may eliminated the use of a thick needle. It is expected that if the visibility is heightened with the use of an echogenic needle, a decrease in the performance time may result as well as an improvement in the quality of the block.

A limitation of this study was that it could not a completely blinded method. First, although another researcher who did not participate in the anesthesia evaluated the nerve functions, this researcher was not blinded to which group the patient was allocated to. Since evaluation of nerve function started immediately after the block, it was not possible to remove the traces indicating the group type such as the application of Betadine. Even though this could be removed, the puncture wound from the needle is visible. To prevent this, all patients would have to have puncture wounds for both approaches, but this would be unethical. Secondly, the anesthetist performing the procedure also knows the patients' group while applying the Betadine and preparing for the block; therefore, there is a possibility of bias during these procedures which could affect the preparation time according to the group. One improvement to the experimental design could be to measure the preparation time separately.

Tran et al. compared the supraclavicular approach, infraclavicular approach, and axillary approach for ultrasoundguided BPB [4]. In their study, the performance time was also shorter for the infraclavicular approach compared to the axillary approach, but there were no differences in the onset times. The difference between ours and their study was that various members from residents to fellows and even staff anesthesiologists participated as the anesthetist performing the block. Further, the evaluation of the nerve block was not done immediately after the procedure and a catheter was inserted for pain control after the operation. This is considered to have affected the measurement of the onset time. On the other hand, 
the difference in the onset time in our study can also be due to measurement error caused by the delay in the performance time of the axillary approach.

In conclusion, ultrasound-guided infraclavicular BPB can reduce the performance time compared to ultrasound-guided axillary block and there was no significant difference in the success rate. Since the block onset time was longer, there were no differences found between the total anesthetic times of both approaches.

\section{References}

1. Minville V, Amathieu R, Luc N, Gris C, Fourcade O, Samii K, et al. Infraclavicular brachial plexus block versus humeral approach: comparison of anesthetic time and efficacy. Anesth Analg 2005; 101: 1198-201.

2. Wedel DJ, Horlocker TT. Nerve blocks. In: Miller's Anesthesia. 7th ed. Edited by Ronald DM: Philadelphia, Churchill Livingstone. 2010, pp 1639-75.

3. Kapral S, Krafft P, Eibenberger K, Fitzgerald R, Gosch M, Weinstabl C. Ultrasound-guided supraclavicular approach for regional anesthesia of the brachial plexus. Anesth Analg 1994; 78: 507-13.

4. Tran de QH, Russo G, Muñoz L, Zaouter C, Finlayson RJ. A prospective, randomized comparison between ultrasound-guided supraclavicular, infraclavicular, and axillary brachial plexus blocks. Reg Anesth Pain Med 2009; 34: 366-71.

5. Ootaki C, Hayashi H, Amano M. Ultrasound-guided infraclavicular brachial plexus block: an alternative technique to anatomical landmark-guided approaches. Reg Anesth Pain Med 2000; 25: 6004.

6. Sites BD, Beach ML, Spence BC, Wiley CW, Shiffrin J, Hartman GS, et al. Ultrasound guidance improves the success rate of a perivascular axillary plexus block. Acta Anaesthesiol Scand 2006; 50: 678-84.

7. Tran de QH, Clemente A, Tran DQ, Finlayson RJ. A comparison between ultrasound-guided infraclavicular block using the "double bubble" sign and neurostimulation-guided axillary block. Anesth Analg 2008; 107: 1075-8.

8. Han JU, Jung JK, Lim HK, Lee JH, Seo CH, Shinn HK. Usefulness of ultrasound-guided infraclavicular brachial plexus block: a comparison with nerve stimulation method. Korean J Anesthesiol 2008; 55: 436-40. 